\title{
Highly Tunable Colloidal Perovskite Nanoplatelets through Variable Cation, Metal, and Halide Composition
}

\author{
Mark C. Weidman, ${ }^{\dagger}$ Michael Seitz, ${ }^{\dagger}$ Samuel D. Stranks, ${ }^{\ddagger} \S$ and William A. Tisdale ${ }^{* \dagger}$ \\ ${ }^{\dagger}$ Department of Chemical Engineering and ${ }^{\ddagger}$ Research Laboratory of Electronics, Massachusetts Institute of Technology, 77 \\ Massachusetts Avenue, Cambridge, Massachusetts 02139, United States \\ ${ }^{\S}$ Cavendish Laboratory, University of Cambridge, JJ Thomson Avenue, Cambridge CB3 0HE, United Kingdom
}

\author{
Supporting Information
}

\begin{abstract}
Colloidal perovskite nanoplatelets are a promising class of semiconductor nanomaterials-exhibiting bright luminescence, tunable and spectrally narrow absorption and emission features, strongly confined excitonic states, and facile colloidal synthesis. Here, we demonstrate the high degree of spectral tunability achievable through variation of the cation, metal, and halide composition as well as nanoplatelet thickness. We synthesize nanoplatelets of the form $L_{2}\left[\mathrm{ABX}_{3}\right]_{n-1} \mathrm{BX}_{4}$, where $\mathrm{L}$ is an organic ligand (octylammonium, butylammo-

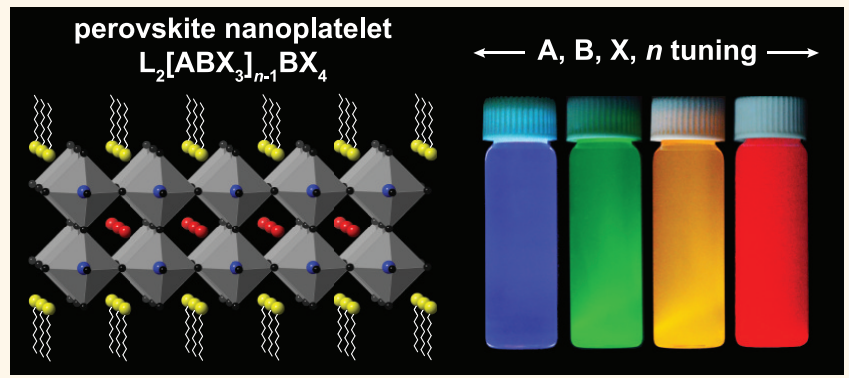
nium), $\mathrm{A}$ is a monovalent metal or organic molecular cation (cesium, methylammonium, formamidinium), $\mathrm{B}$ is a divalent metal cation (lead, tin), $\mathrm{X}$ is a halide anion (chloride, bromide, iodide), and $n-1$ is the number of unit cells in thickness. We show that variation of $n, \mathrm{~B}$, and $\mathrm{X}$ leads to large changes in the absorption and emission energy, while variation of the A cation leads to only subtle changes but can significantly impact the nanoplatelet stability and photoluminescence quantum yield (with values over 20\%). Furthermore, mixed halide nanoplatelets exhibit continuous spectral tunability over a $1.5 \mathrm{eV}$ spectral range, from 2.2 to $3.7 \mathrm{eV}$. The nanoplatelets have relatively large lateral dimensions (100 $\mathrm{nm}$ to $1 \mu \mathrm{m}$ ), which promote self-assembly into stacked superlattice structures - the periodicity of which can be adjusted based on the nanoplatelet surface ligand length. These results demonstrate the versatility of colloidal perovskite nanoplatelets as a material platform, with tunability extending from the deep-UV, across the visible, into the near-IR. In particular, the tin-containing nanoplatelets represent a significant addition to the small but increasingly important family of lead- and cadmium-free colloidal semiconductors.
\end{abstract}

KEYWORDS: perovskite, nanoplatelet, metal halide, colloid, 2D, quantum confinement

ecent progress in metal halide perovskite solar cells has highlighted many desirable properties of these semiconductor materials, including long charge carrier diffusion length, ease of fabrication, and low trap state density. ${ }^{1-7}$ The rapid advancement in perovskite solar cells has also led to a renewed interest in nanostructured and colloidal perovskite-based materials. ${ }^{8-16}$ While low-dimensional, layered perovskite materials have been studied in the past, ${ }^{17-23}$ bright and colloidally stable versions of these materials have only recently been developed. , $^{8,11,24-26}$ Perovskite nanoplatelets are particularly interesting because they exhibit strong quantum confinement effects, which enable thickness-dependent property tuning. Furthermore, Quan et al. and Tsai et al. have demonstrated that layered $2 \mathrm{D}$ perovskite solar cells exhibit enhanced resistance to air and water exposure as compared to their bulk counterparts, likely a result of surface passivation provided by ligand species. ${ }^{27,28}$ Other recent studies have shown the potential for thickness-controlled perovskite materials for use in efficient light-emitting diodes. ${ }^{29,30}$

The colloidal synthesis of methylammonium lead bromide $\left(\mathrm{MAPbBr}_{3}\right)$ perovskite nanoparticles was first reported by Schmidt et al., producing a mixture of bulk-like nanoparticles and quantum-confined nanoplatelets. ${ }^{8}$ Tyagi et al. were able to isolate the nanoplatelets and demonstrated their thicknessdependent absorption and emission, ${ }^{10}$ with Sichert et al. showing that these nanoplatelets could be directly synthesized with control over nanoplatelet thickness. ${ }^{11}$ Other recent works have extended the synthetic capabilities to include cesium lead halide $\left(\mathrm{CsPbX}_{3}\right)$ nanoplatelets ${ }^{31-34}$ and methylammonium lead chloride and iodide $\left(\mathrm{MAPbCl}_{3}, \mathrm{MAPbI}_{3}\right)$ nanoplatelets. ${ }^{35-38}$

Received: May 26, 2016

Accepted: July 29, 2016

Published: July 29, 2016 
As bulk perovskites can be described by the formula $\mathrm{ABX}_{3}$, where $\mathrm{A}$ is a cation, $\mathrm{B}$ is a metal, and $\mathrm{X}$ is a halide, perovskite nanoplatelets can similarly be described by the formula $\mathrm{L}_{2}\left[\mathrm{ABX}_{3}\right]_{n-1} \mathrm{BX}_{4}$. In this case, we have chosen $\mathrm{L}$ to represent the ligand species which both gives the nanoplatelet colloidal stability and limits growth in one dimension of the nanoplatelet. Here, the $n-1$ term represents the thickness of the nanoplatelet in terms of the bulk unit cell, with $n=2$ corresponding to a complete perovskite unit cell and $n=1$ corresponding to an incomplete perovskite structure that lacks the A cation altogether.

In this work, we focus on the thinnest perovskite nanoplatelets, $n=1$ and $n=2$, which exhibit the greatest degree of quantum confinement and the greatest deviation from bulk properties. ${ }^{39}$ By taking advantage of quantum confinement effects, it is possible to blue shift the absorption and emission energies by $\sim 0.6 \mathrm{eV}$ relative to their bulk values. This shift enables the typically more emissive bromide and iodide-based perovskites (as compared to chloride) ${ }^{49}$ to cover a majority of the visible range, including excellent performance in the blue region of the spectrum. Furthermore, we find that the $n=1$ and 2 nanoplatelets can most reliably be synthesized with single-thickness ensemble purity, which results in the narrowest absorption and emission line widths. Despite these advantages, a compositionally flexible, unified synthesis method for $n=1$ and $n=2$ nanoplatelet has yet to be developed. In this study, we synthesize colloidally stable $n=1$ and $n=2$ perovskite nanoplatelets using a facile nonsolvent crystallization process and show that the $\mathrm{A}, \mathrm{B}$, and $\mathrm{X}$ components can be changed across a wide range of chemical species. This, in conjunction with thickness tuning, allows for modification of the absorption and emission properties from the deep-UV, throughout the visible, and into the near-IR. We show that changes to the thickness $(n)$, metal (B), and halide (X) lead to large changes in the absorption and emission wavelength. On the other hand, the cation (A) species has a small effect on the nanoplatelet absorption and emission energy yet can have a large effect on the stability of the nanoplatelet and the photoluminescence quantum yield (PLQY). We found that formamidinium is an excellent cation for nanoplatelets, with narrower emission and increased PLQY over methylammonium. Furthermore, we demonstrate that using mixtures of the halide component is a viable method of continuously tuning the properties between the pure component states. Lastly, we have synthesized nanoplatelets using tin as the metal component, which is a critical step toward lead- and cadmium-free luminescent nanoparticles. With this study, we aim to demonstrate the exceptional tunability of perovskite nanoplatelets and to highlight some of the most promising compositions that could find application in high-performance light-emitting diodes.

\section{RESULTS AND DISCUSSION}

Nanoplatelet Structure. The bulk perovskite unit cell, outlined in Figure 1a as a white cube, has the formula $\mathrm{ABX}_{3}$. Typically, $A$ is a cation with +1 oxidation state, $B$ is a metal with +2 oxidation state, and $\mathrm{X}$ is a halide with -1 oxidation state. Perovskite nanoplatelets, which are confined dimensionally in one direction, can be described using the formula $\mathrm{L}_{2}\left[\mathrm{ABX}_{3}\right]_{n-1} \mathrm{BX}_{4}$, where $\mathrm{L}$ represents a longer chain cation which gives the nanoplatelet colloidal stability but also serves to inhibit growth of the nanoplatelet, as it is too large to fit within the unit cell geometry. ${ }^{40,41}$ Nanoplatelets with thicknesses $n=1$ a

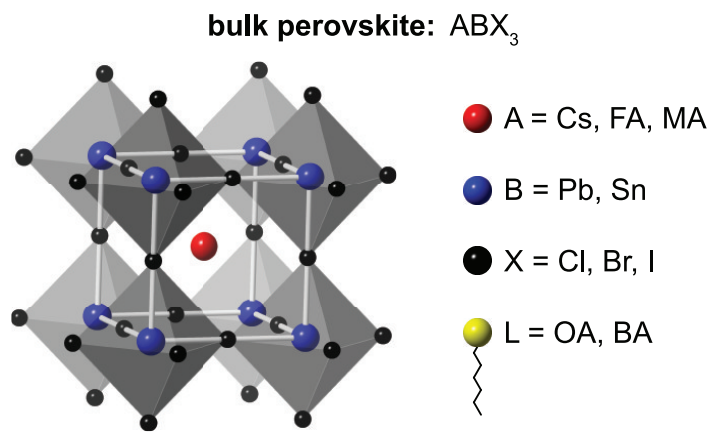

b nanoplatelet: $\mathrm{L}_{2}\left[\mathrm{ABX}_{3}\right]_{n-1} \mathrm{BX}{ }_{4}$

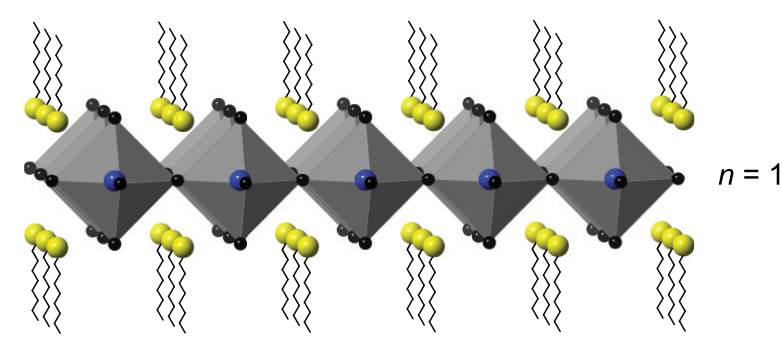

C

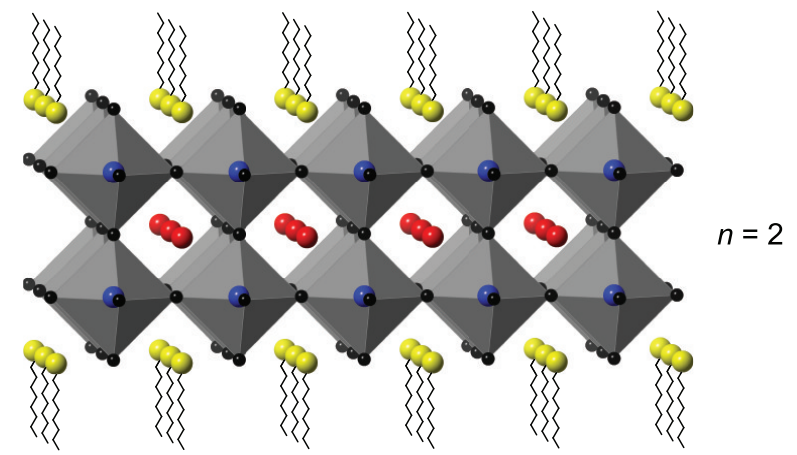

Figure 1. (a) Bulk perovskite unit cell (white cube) and the chemical species used for $A, B, X$, and $L$ in this study. (b,c) Perovskite nanoplatelets of thicknesses $n=1$ and $n=2$, where $n$ represents the layers of metal halide octahedra and $n-1$ represents the number of complete unit cells incorporated in the nanoplatelet thickness.

and $n=2$ are depicted in Figure $1 \mathrm{~b}, \mathrm{c}$, respectively. Using this notation, $n$ represents the number of metal halide octahedra layers present in the nanoplatelet. The number $n-1$ represents how many complete perovskite unit cells fit within the thickness of the nanoplatelet. Hence, for $n=1$ nanoplatelets, there is no cation species (A) contained within the structure.

In this study, we have synthesized colloidal perovskite nanoplatelets $(n=1$ and $n=2)$ through the nonsolvent crystallization process described in the Experimental Methods section. For ease of writing nanoplatelet compositions, we use the following abbreviations throughout to represent different chemical species. A: cesium $=\mathrm{Cs}$, formamidinium $=\mathrm{FA}$, methylammonium $=\mathrm{MA}$. B: lead $=\mathrm{Pb}$, tin $=\mathrm{Sn}$. X: chloride $=$ $\mathrm{Cl}$, bromide $=\mathrm{Br}$, iodide $=\mathrm{I}$. $\mathrm{L}$ : butylammonium $=\mathrm{BA}$, octylammonium $=\mathrm{OA}$. In all cases, the ligand species $\mathrm{L}$ has been a 50/50 mixture of OA and BA.

We have targeted $n=1$ and $n=2$ thicknesses because they can be made with the best thickness selectivity, as compared to $n=3,4,5$, etc. (see Figure S7), which tend to result in mixtures of nanoplatelet thickness. For instance, synthesis of $n=1$ nanoplatelets does not include the A cation, and because the $\mathrm{L}$ 
cation is too large to pack within the $\mathrm{ABX}_{3}$ unit cell, the nanoplatelet thickness is naturally confined to one layer of metal halide octahedra. Nanoplatelets with thickness $n=2$ face the challenge that growth can exceed past the intended thickness, leading to more bulk-like properties. We found that simply using the stoichiometry dictated by $n=2$ ( 2 parts LX, 2 parts $\mathrm{BX}_{2}, 1$ part $\mathrm{AX}$ ) forms $n=2$ nanoplatelets but also significant quantities of thicker nanoplatelets (see Figure S8). However, by increasing the relative concentration of the ligand (L), which inhibits growth in the confined direction, we were able to synthesize pure $n=2$ nanoplatelet dispersions with excellent thickness homogeneity.

In Figure 2, we show representative transmission electron microscopy (TEM) images for the $n=1$ and $n=2$ nanoplatelets synthesized using the nonsolvent crystallization method for all of the B and X configurations studied here (A was FA for all $n=2$ nanoplatelets). The images demonstrate the flexible nature of the synthesis, which yields similar products regardless of the chemical identities of $\mathrm{A}, \mathrm{B}$, and $\mathrm{X}$. The nanoplatelets have lateral dimensions on the order of several hundred nanometers, even reaching micron scale in some cases (Figure S11). The nanoplatelets typically have the shape of rectangles with rounded corners; however, in some cases, we have observed sharper corners (Figure S11). As others have reported, ${ }^{8,10,11}$ we also observed dark clusters present on the surfaces of nanoplatelets when imaged at higher magnifications (Figure S12). However, their presence was not consistent across all nanoplatelets, and their exact identity is not fully understood at this time.

X-ray diffraction (XRD) measurements of drop-cast thin films of nanoplatelets showed periodic diffraction at low angles, indicative of nanoplatelet stacking. Typical patterns for $n=1$ and $n=2$ nanoplatelets are shown in Figure $3 \mathrm{a}$, and we find these to be representative of all samples studied (see Figures S15-S17). The reflections from nanoplatelet stacks are denoted by diamonds above select peaks. We hypothesize that the strong reflections from these superstructures are due to the large lateral dimensions of the nanoplatelets synthesized here, which forces them to lie flat when deposited in a thin film (see Figure S13). Nevertheless, for $n=2$ nanoplatelets, we also observe the typical peaks resulting from atomic plane reflections of the perovskite at 14.9 and $29.9^{\circ}$ [the (100) and (200) planes], indicated by black circles above the peaks. ${ }^{46} \mathrm{We}$ note that mixing the nanoplatelets with silica particles prior to deposition can disrupt the stacking to more clearly show the atomic plane reflections (see Figure S18), confirming the perovskite atomic structure. The peaks from nanoplatelet stacking are at regular intervals of 5.1 and $3.9^{\circ}$ for the $n=1$ and $n=2$ nanoplatelets, respectively. These periodicities correspond to average spacing between layered nanoplatelets of 1.7 $\mathrm{nm}$ for $n=1$ and $2.3 \mathrm{~nm}$ for $n=2$ nanoplatelets. We find that the spacing is quite consistent regardless of the chemical composition of the nanoplatelets (see Table S4).

The $n=1$ nanoplatelets stack with an average spacing of 1.7 $\mathrm{nm}$ over the different compositions studied here (Table S4). As these nanoplatelets were made without any cation (A) species which can fit within the perovskite unit cell, we know the nanoplatelet consists of a single metal halide octahedra layer, which is $\sim 0.6 \mathrm{~nm} .{ }^{10,42}$ Therefore, the ligands occupy a space of $\sim 1.1 \mathrm{~nm}$. The nanoplatelet ligands are an equimolar mixture of octylammonium $(\sim 1.0 \mathrm{~nm})$ and butylammonium $(\sim 0.5 \mathrm{~nm})$, and so this length is consistent with some ligand interdigitation between neighboring nanoplatelets. The physical picture is
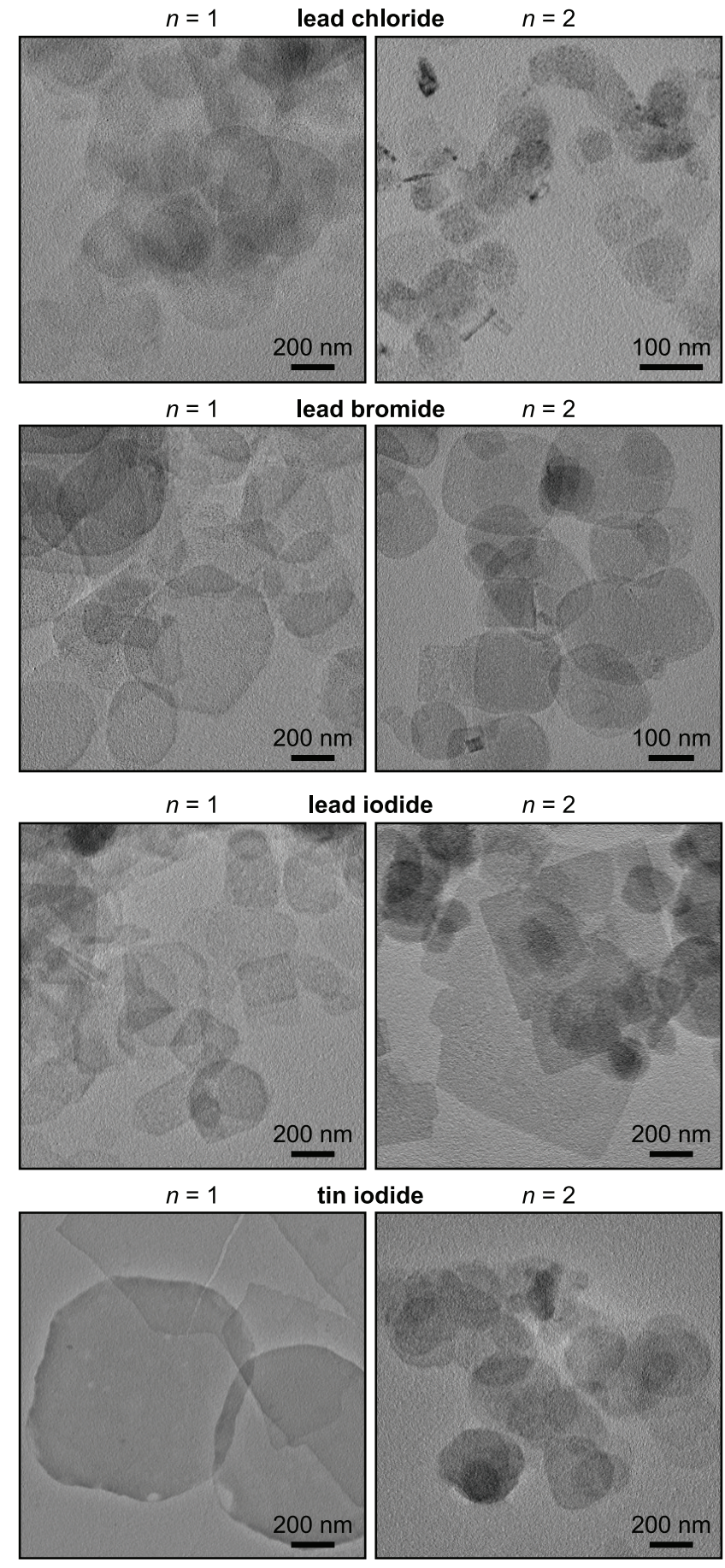

Figure 2. TEM images of $n=1$ and $n=2$ nanoplatelets, $\mathrm{L}_{2}\left[\mathrm{ABX}_{3}\right]_{n-1} \mathrm{BX}_{4}$, with $\mathrm{A}=\mathrm{FA}, \mathrm{B}=\mathrm{Pb}$ or $\mathrm{Sn}$, and $\mathrm{X}=\mathrm{Cl}$, Br, or $\mathrm{I}$.

depicted in Figure $3 \mathrm{~b}$. The $n=2$ nanoplatelets have an average spacing of $2.3 \mathrm{~nm}$ between stacks. Assuming the ligands again occupy a space of $\sim 1.1 \mathrm{~nm}$, this means the perovskite part of the nanoplatelet is $\sim 1.2 \mathrm{~nm}$ thick, or 2 metal halide octahedra layers thick, with one full unit cell contained within. This depiction is shown in Figure 3c. Using these XRD measurements, we can confirm that the $n=2$ nanoplatelets are in fact two layers of metal halide octahedra with one full unit cell incorporated and that the nanoplatelets have not grown into thicker analogues $(n>2)$. 
a

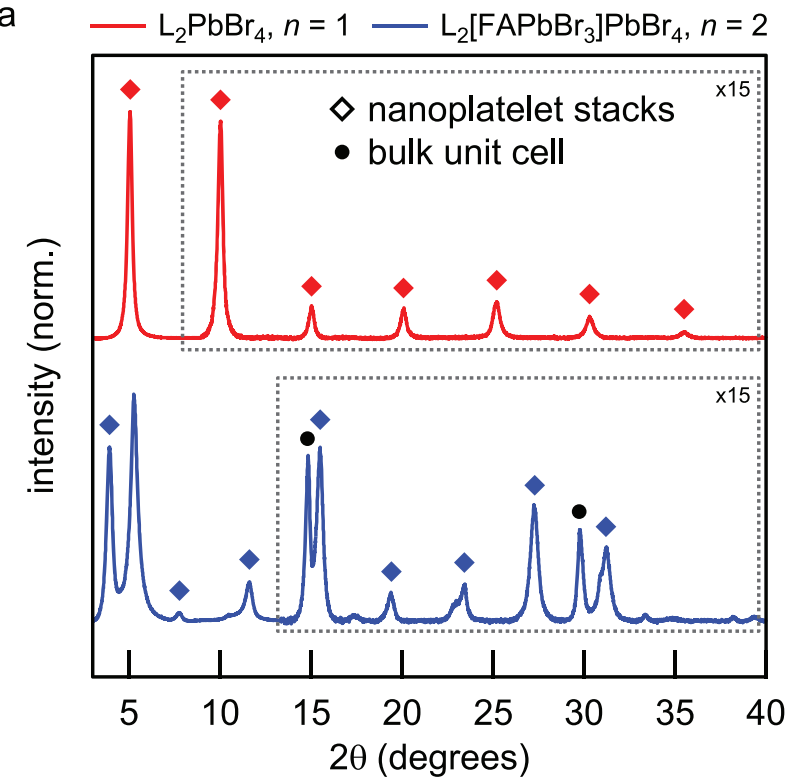

b

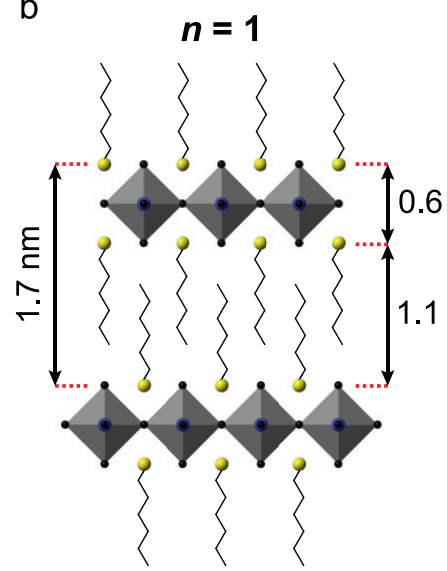

C

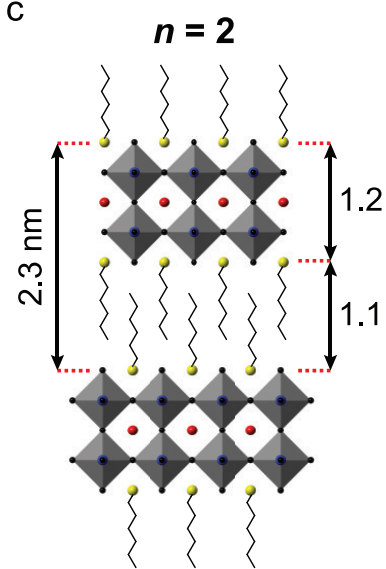

Figure 3. (a) XRD patterns for $n=1\left(\mathrm{~L}_{2} \mathrm{PbBr}_{4}\right)$ and $n=2$ $\left(\mathrm{L}_{2}\left[\mathrm{FAPbBr}_{3}\right] \mathrm{PbBr}_{4}\right)$ nanoplatelets showing periodic reflections from nanoplatelet stacks which form in thin film samples (indicated by diamonds). Black circles indicate peaks corresponding to the bulk perovskite unit cell. ${ }^{10}$ Sections of the XRD patterns have been scaled to better show the peaks at larger angles. The XRD patterns indicate a stacking distance of 1.7 and $2.3 \mathrm{~nm}$ for $n=1$ and $n=2$, respectively, regardless of chemical composition. (b,c) Schematic representations of the nanoplatelet stacks and relevant distances.

We hypothesize that the extensive nanoplatelet stacking observed through $\mathrm{XRD}$ is mainly due to their large lateral dimensions-nanoplatelets synthesized here tend to have lateral dimensions of $100 \mathrm{~nm}$ to $1 \mu \mathrm{m}$, whereas those synthesized at elevated temperatures tend to be $<100$ nm. ${ }^{32,35}$ This large size causes the nanoplatelets to lie flat on the substrate and overlap with each other in a well-defined manner. As a result, we observe extensive superlattices of stacked nanoplatelets with preferred orientation along the (h00) direction. To further study these superlattices, we synthesized $\mathrm{L}_{2} \mathrm{PbBr}_{4}$ nanoplatelets with different surface ligand lengths. The XRD patterns from the drop-cast films of these samples are presented in Figure 4. We found that the periodicity of reflections from the superlattice (marked with diamonds above each peak) changed according to the ligands used to synthesize the nanoplatelets. BA ligands led to the shortest stacking distance of $1.3 \mathrm{~nm}$ between nanoplatelets,

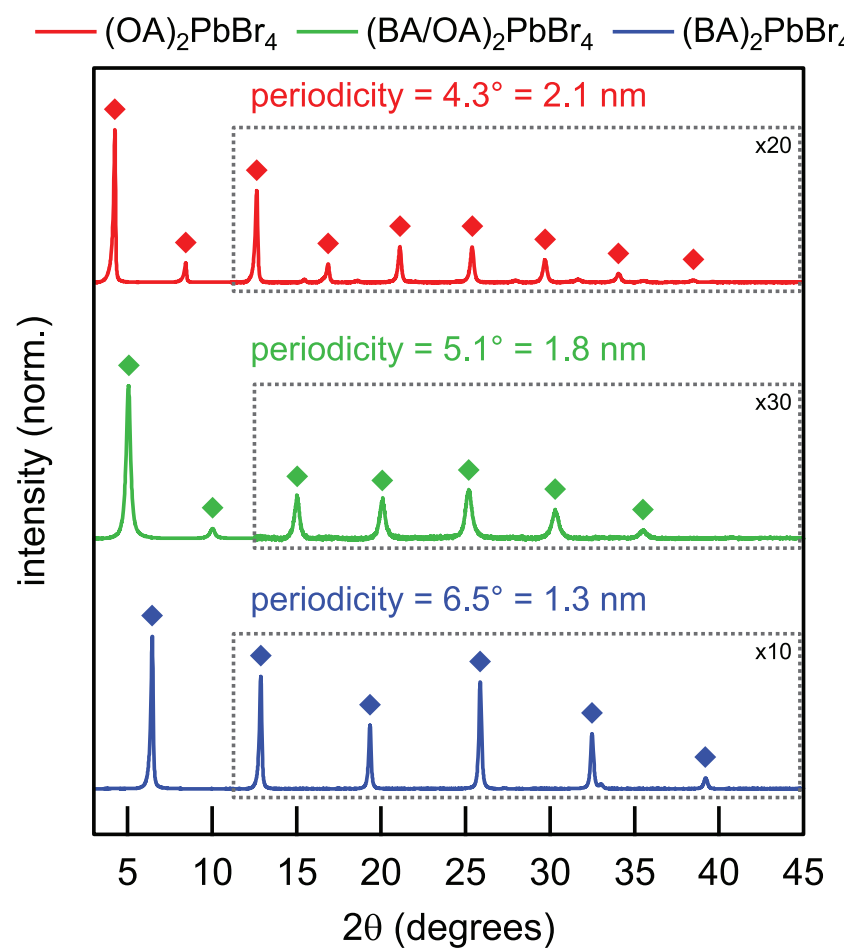

Figure 4. $\mathrm{XRD}$ patterns for $\mathrm{L}_{2} \mathrm{PbBr}_{4}$ nanoplatelets where the ligand species, $\mathrm{L}$, was either octylammonium, butylammonium, or an equimolar mixture of the two (BA/OA), which results in changes to the nanoplatelet superlattice stacking distance.

while OA ligands led to a stacking distance of $2.1 \mathrm{~nm}$. An equimolar mixture of $\mathrm{BA}$ and $\mathrm{OA}$ fell between these two values, skewing slightly more toward the pure OA value. The ability to tune the superlattice stacking distance could lead to improved control of energy transfer rates between stacks of nanoplatelets and a better understanding of how these nanoplatelets behave in close proximity.

Varying the Metal (B) and Halide (X) Components. As with their bulk counterparts, we find the $\mathrm{A}, \mathrm{B}$, and $\mathrm{X}$ components of the nanoplatelets to be highly tunable, providing a robust strategy for achieving a desired absorption and photoluminescence peak position. We have explored many species for A (Cs, MA, FA), B ( Pb, Sn), and X ( $\mathrm{Cl}, \mathrm{Br}, \mathrm{I})$. In Figure 5, we present the effects of changing the metal (B) and halide $(X)$ species, which result in large changes to the nanoplatelet absorption and emission peaks. Starting with the $\mathrm{X}$ species, we find that the $n=1$ and $n=2$ nanoplatelets of $\mathrm{L}_{2}\left[\mathrm{FAPbCl}_{3}\right]_{n-1} \mathrm{PbCl}_{4}$ have peak absorption at 3.71 and 3.50 $\mathrm{eV}$, respectively. The emission from these nanoplatelets was not measured, as their absorption is higher in energy than our 365 $\mathrm{nm}$ excitation source. By substituting bromide for chloride, the peak absorption/emission for $\mathrm{L}_{2}\left[\mathrm{FAPbBr}_{3}\right]_{n-1} \mathrm{PbBr}_{4} n=1$ and $n=2$ nanoplatelets is shifted lower in energy to $3.12 / 3.08$ and $2.86 / 2.82 \mathrm{eV}$, respectively. Once again, substituting iodide in place of bromide, the peak absorption/emission for $\mathrm{L}_{2}\left[\mathrm{FAPbI}_{3}\right]_{n-1} \mathrm{PbI}_{4} n=1$ and $n=2$ nanoplatelets is shifted to $2.45 / 2.41$ and $2.19 / 2.16 \mathrm{eV}$, respectively. These values are compiled in Table 1 . Thus, by changing the halide, it is possible to go from deep-UV absorption (chlorides) to violet/blue emission (bromides) to green/yellow emission (iodides). In a similar fashion, by substituting $\mathrm{Sn}$ for $\mathrm{Pb}$, we see again that the peak absorption/emission for $\mathrm{L}_{2}\left[\mathrm{FASnI}_{3}\right]_{n-1} \mathrm{SnI}_{4} n=1$ and $n=$ 2 nanoplatelets is shifted to $2.05 / 1.97$ and $1.83 / 1.80 \mathrm{eV}$, 


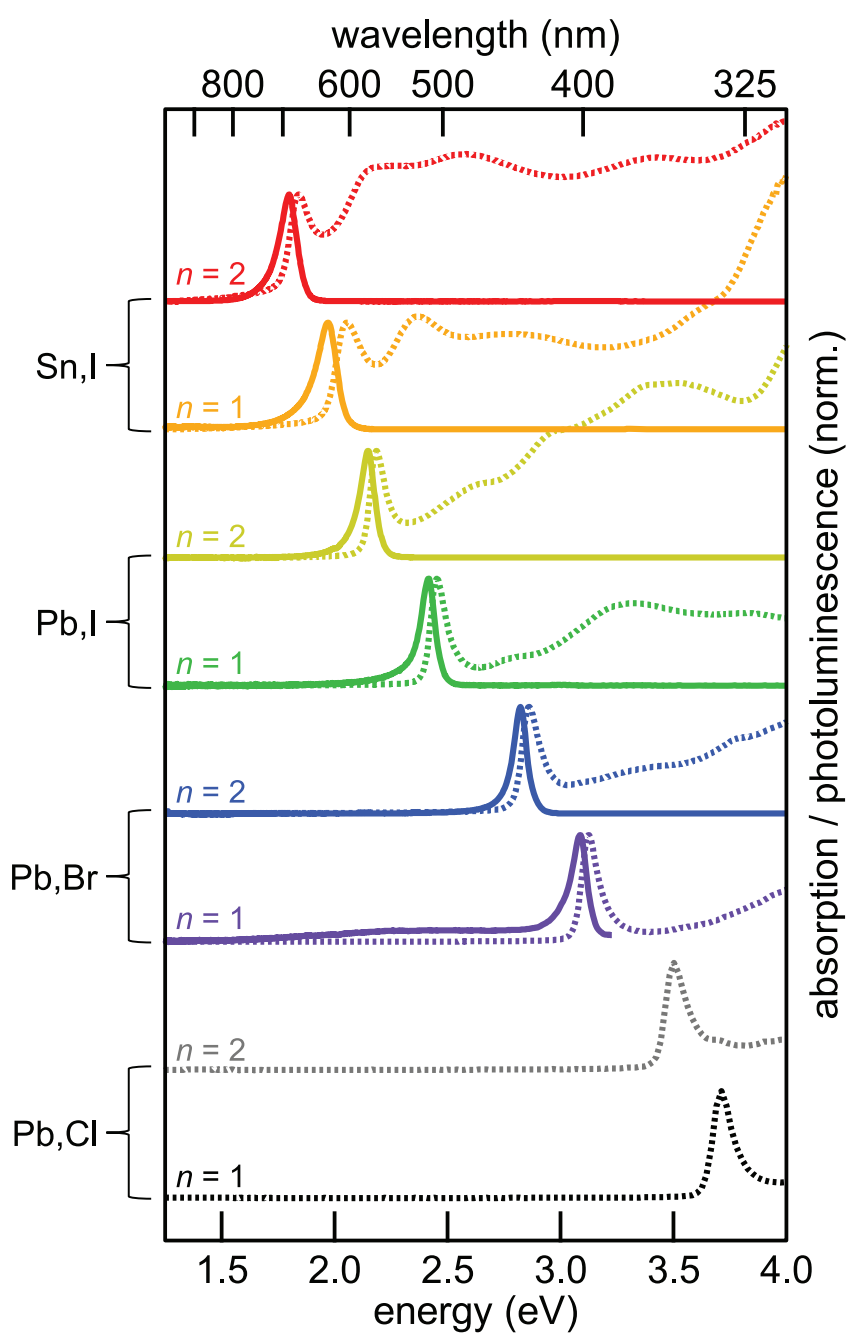

Figure 5. (a) Solution phase absorption (dotted lines) and photoluminescence (solid lines) spectra for $n=1$ and $n=2$ nanoplatelets in toluene, highlighting the changes which occur when the halide $(\mathrm{X})$ is changed from $\mathrm{Cl}$ to $\mathrm{Br}$ to $\mathrm{I}$ and when the metal (B) is changed from $\mathrm{Pb}$ to $\mathrm{Sn}$.

respectively. As demonstrated in Figure 5, by selection of the metal (B) and halide (X), it is possible to tune the absorption/ emission of $n=1$ and $n=2$ nanoplatelets to span the entire visible region of the spectrum.

Varying the Cation (A) Component. As with the B and X components, we can also use a variety of species for the cation (A) in $n=2$ nanoplatelets. However, we found that changing the chemical identity of the cation only alters the absorption and emission energy of the nanoplatelet slightly. ${ }^{43,44}$ In Figure $6 \mathrm{a}$, we present the absorption and emission of $n=2$ nanoplatelets, $\mathrm{L}_{2}\left[\mathrm{APbBr}_{3}\right] \mathrm{PbBr}_{4}$, where $\mathrm{A}$ has been varied between Cs, MA, and FA. As the size of the cation increases (cation size: Cs < MA < FA), ${ }^{41}$ the absorption peak shifts to slightly lower energies, in $\sim 20 \mathrm{meV}$ increments (see Table 1). This cation-dependent energy shift is also observed in the bulk phase and follows the same size-dependent trend. ${ }^{45,46}$ We show in Figure $6 \mathrm{~b}$ that similar behavior is observed for $\mathrm{L}_{2}\left[\mathrm{APbI}_{3}\right] \mathrm{PbI}_{4}$ nanoplatelets, though the difference between MA and FA is less pronounced. See Figure S10 for the absorption spectra highlighting the differences between MA and FA in leadchloride-based $n=2$ nanoplatelets.
Table 1. Summary of Absorption and Emission Properties of Perovskite Nanoplatelets $\left(\mathrm{L}_{2}\left[\mathrm{ABX}_{3}\right]_{n-1} \mathrm{BX}_{4}\right)$ and Bulk, Polycrystalline Perovskite $\left(\mathrm{ABX}_{3}\right)$ Phase (Denoted $n=\infty$ )

\begin{tabular}{|c|c|c|c|c|c|c|}
\hline \multirow[b]{2}{*}{ formula } & \multirow[b]{2}{*}{$n$} & \multicolumn{2}{|c|}{ absorption $^{a}$} & \multicolumn{3}{|c|}{ emission } \\
\hline & & $\mathrm{nm}$ & $\mathrm{eV}$ & $\mathrm{nm}$ & $\mathrm{eV}$ & $\begin{array}{l}\text { fwhm } \\
(\mathrm{meV})\end{array}$ \\
\hline $\mathrm{L}_{2} \mathrm{PbCl}_{4}$ & 1 & 334 & 3.71 & & & \\
\hline $\mathrm{L}_{2}\left[\mathrm{MAPbCl}_{3}\right] \mathrm{PbCl}_{4}$ & 2 & 348 & 3.57 & & & \\
\hline $\mathrm{L}_{2}\left[\mathrm{FAPbCl}_{3}\right] \mathrm{PbCl}_{4}$ & 2 & 354 & 3.50 & & & \\
\hline $\mathrm{MAPbCl}_{3}^{49}$ & $\infty$ & 390 & 3.18 & 413 & 3.00 & \\
\hline $\mathrm{L}_{2} \mathrm{PbBr}_{4}$ & 1 & 398 & 3.12 & 403.2 & 3.08 & 89 \\
\hline $\mathrm{L}_{2}\left[\mathrm{CsPbBr}_{3}\right] \mathrm{PbBr}_{4}$ & 2 & 429 & 2.89 & 433.2 & 2.86 & 81 \\
\hline $\mathrm{L}_{2}\left[\mathrm{MAPbBr}_{3}\right] \mathrm{PbBr}_{4}$ & 2 & 431 & 2.88 & 437.3 & 2.83 & 89 \\
\hline $\mathrm{L}_{2}\left[\mathrm{FAPbBr}_{3}\right] \mathrm{PbBr}_{4}$ & 2 & 434 & 2.86 & 439 & 2.82 & 71 \\
\hline $\mathrm{CsPbBr}_{3}^{50,51}$ & $\infty$ & 525 & 2.36 & 527 & 2.35 & \\
\hline $\mathrm{MAPbBr}_{3}^{52}$ & $\infty$ & 530 & 2.34 & 540 & 2.30 & \\
\hline $\mathrm{FAPbBr}_{3}{ }^{52}$ & $\infty$ & 549 & 2.26 & 548 & 2.26 & \\
\hline $\mathrm{L}_{2} \mathrm{PbI}_{4}$ & 1 & 506 & 2.45 & 512.8 & 2.42 & 76 \\
\hline $\mathrm{L}_{2}\left[\mathrm{CsPbI}_{3}\right] \mathrm{PbI}_{4}$ & 2 & 553 & 2.24 & 561.1 & 2.21 & 79 \\
\hline $\mathrm{L}_{2}\left[\mathrm{MAPbI}_{3}\right] \mathrm{PbI}_{4}$ & 2 & 566 & 2.19 & 573.9 & 2.16 & 83 \\
\hline $\mathrm{L}_{2}\left[\mathrm{FAPbI}_{3}\right] \mathrm{PbI}_{4}$ & 2 & 566 & 2.19 & 575 & 2.16 & 76 \\
\hline $\mathrm{CsPbI}_{3}{ }^{12,46}$ & $\infty$ & 717 & 1.73 & 714 & 1.74 & \\
\hline $\mathrm{MAPbI}_{3}{ }^{46,53,54}$ & $\infty$ & 789 & 1.57 & 783 & 1.58 & \\
\hline $\mathrm{FAPbI}_{3}^{46,53}$ & $\infty$ & 838 & 1.48 & 810 & 1.53 & \\
\hline $\mathrm{L}_{2} \mathrm{SnI}_{4}$ & 1 & 604 & 2.05 & 628.2 & 1.97 & 104 \\
\hline $\mathrm{L}_{2}\left[\mathrm{FASnI}_{3}\right] \mathrm{SnI}_{4}$ & 2 & 674 & 1.84 & 689 & 1.80 & 93 \\
\hline $\mathrm{FASnI}_{3}{ }^{55,56}$ & $\infty$ & 880 & 1.41 & & & \\
\hline
\end{tabular}

${ }^{a}$ Nanoplatelet absorption is based on the peak location; bulk absorption is the absorption onset as calculated by the Tauc plot.

While changes to the cation (A) produce subtle variations in the resulting absorption and emission energy of the nanoplatelets, there are more significant implications in terms of spectral quality and PLQY. For both the bromide and iodide nanoplatelets, the fwhm of the emission peaks follows the trend of FA $<$ Cs $<$ MA, as listed in Table 1 . In particular, we find that MA generally leads to a more broadened emission peak $(89,83$ $\mathrm{meV})$ than either FA $(71,76 \mathrm{meV})$ or $\mathrm{Cs}(81,79 \mathrm{meV})$. The absorption peak width (hwhm to the red side of peak) of MA nanoplatelets also tends to be larger than the widths of FA and Cs nanoplatelets, which were found to have similar absorption line widths. Furthermore, $n=2$ nanoplatelets synthesized with Cs tend to evolve into thicker nanoplatelets more readily than those made with MA and FA. We attribute this to the small size of the cation, which may more easily enable postsynthesis structural rearrangement within the nanoplatelet. Furthermore, we find that the PLQY of nanoplatelets synthesized with FA, as compared to MA, tends to be higher, as listed in Table 2. In particular, the use of FA over MA provides a large boost in the PLQY of $\mathrm{L}_{2}\left[\mathrm{APbBr}_{3}\right] \mathrm{PbBr}_{4}$ nanoplatelets, from around $6 \%$ with MA to $22 \%$ with FA. The $6 \%$ PLQY achieved with MA is comparable to other reported values in literature-10\% for $n=$ $3 \mathrm{CsPbr}_{3}$ nanoplatelets ${ }^{32}$ and $3 \%$ for $n=2 \mathrm{MAPbBr}_{3}$ nanoplatelets ${ }^{11}$ - while the $22 \%$ PLQY with FA is the highest reported value we are aware of for $n=2$ nanoplatelets. With narrow emission and high PLQY values, FA proved to be an excellent cation for nanoplatelets. Exploration of other cation species may thus be a viable pathway for increasing PLQY and stability of nanoplatelets even further. We also observed that $n$ 


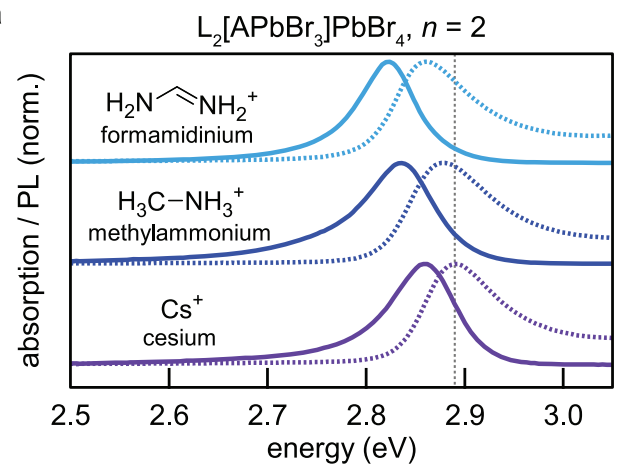

b

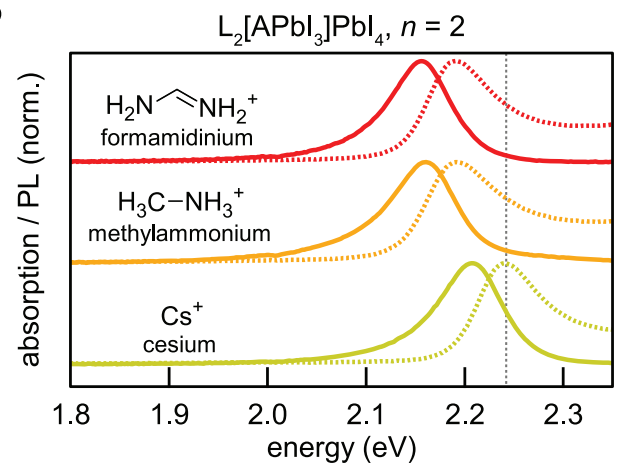

Figure 6. Absorption and photoluminescence spectra for $n=2$ nanoplatelets in toluene with varying cation (A) species for (a) $\mathrm{L}_{2}\left[\mathrm{APbBr}_{3}\right] \mathrm{PbBr}_{4}$ nanoplatelets and (b) $\mathrm{L}_{2}\left[\mathrm{APbI}_{3}\right] \mathrm{PbI}_{4}$ nanoplatelets.

Table 2. Photoluminescence Quantum Yield of Select Nanoplatelets

\begin{tabular}{llc}
\multicolumn{1}{c}{ formula } & $n$ & PLQY (\%) \\
$\mathrm{L}_{2}\left[\mathrm{MAPbBr}_{3}\right] \mathrm{PbBr}_{4}$ & 2 & 6 \\
$\mathrm{~L}_{2}\left[\mathrm{FAPbBr}_{3}\right] \mathrm{PbBr}_{4}$ & 2 & 22 \\
$\mathrm{~L}_{2} \mathrm{PbI}_{4}$ & 1 & 0.2 \\
$\mathrm{~L}_{2}\left[\mathrm{MAPbI}_{3}\right] \mathrm{PbI}_{4}$ & 2 & 1.1 \\
$\mathrm{~L}_{2}\left[\mathrm{FAPbI}_{3}\right] \mathrm{PbI}_{4}$ & 2 & 1.4 \\
$\mathrm{~L}_{2} \mathrm{SnI}_{4}$ & 1 & $0.5^{a}$ \\
$\mathrm{~L}_{2}\left[\mathrm{FASnI}_{3}\right] \mathrm{SnI}_{4}$ & 2 & $2.6^{a}$
\end{tabular}

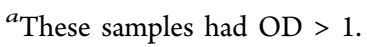

$=1$ nanoplatelets have reduced PLQY compared to that of $n=$ 2 nanoplatelets, which is in agreement with the thicknessdependent trend seen elsewhere. ${ }^{11,32,42}$ Lastly, we note that the tin-based nanoplatelets studied could not be diluted below and optical density of $\sim 1$ (see Supporting Information discussion of tin-based nanoplatelet synthesis), and so the PLQY values likely include absorption/re-emission effects and would be an underestimate of the true PLQY. Despite this, the values for tin-based nanoplatelets are quite high when compared to those of bulk polycrystalline $\mathrm{MASnI}_{3}$ (PLQY was below the detection limit) and $\mathrm{CsSnI}_{3}$ nanoparticles (PLQY $\left.=0.06 \%\right)^{47,48}$

The ability to readily tune the $\mathrm{A}, \mathrm{B}$, and $\mathrm{X}$ components of perovskite nanoplatelets makes them a highly versatile material platform. The absorption peak and emission properties of all the $n, \mathrm{~A}, \mathrm{~B}$, and $\mathrm{X}$ configurations studied here are compiled in Table 1, along with the values for their bulk polycrystalline counterparts. The values for bulk materials have been referenced from literature sources, where the absorption value listed is the absorption onset as calculated by the Tauc ploc, which is typically very close to the emission energy. We also note, as shown in Figure S9, that the nanoplatelet emission peak can be shifted slightly as a function of the excess ligand concentration, which could explain discrepancies throughout literature for $n=2$ peak values and may result in some experimental variation from the listed values. Table 1 highlights the versatility of perovskite nanoplatelets as well as their narrow emission (fwhm 70-90 meV) and small Stokes shifts (30-80 $\mathrm{meV}$ ). Their excellent properties in the blue region of the spectrum, with PLQY up to $22 \%$, are particularly promising, as this can be a challenging region to access using other emissive nanoparticles, such as quantum dots.

Continuous Halide Tunability. We have demonstrated the flexibility of the $\mathrm{A}, \mathrm{B}$, and $\mathrm{X}$ components in perovskite nanoplatelets, which allows for modification of the absorption and emission energies spanning the visible range of the spectrum and into the UV and near-IR. However, the size of these steps is either quite large (when changing the metal or halide species) or quite small (when changing the cation species). For finer control of the absorption and photoluminescence energy, we show in Figure 7 that using mixed
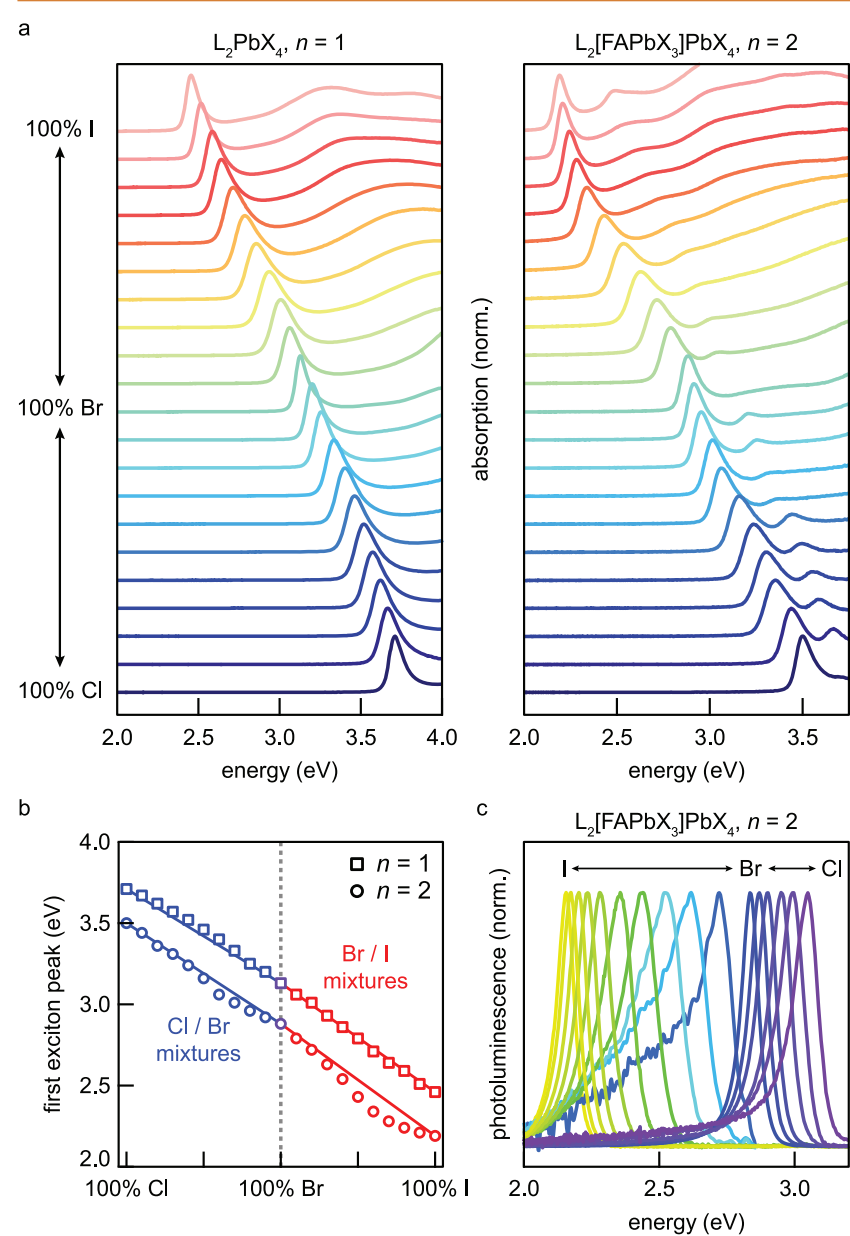

Figure 7. (a) Absorption spectra for $n=1$ and $n=2$ nanoplatelets showing continuous tunability as a function of halide composition. Mixtures were made between $\mathrm{Cl} / \mathrm{Br}$ and $\mathrm{Br} / \mathrm{I}$ in increments of $10 \%$ between the pure compositions. (b) Absorption peak as a function of halide composition. (c) Photoluminescence of $n=2$ nanoplatelets for all $\mathrm{Br} / \mathrm{I}$ mixtures and from $100 \% \mathrm{Br}$ to $50 / 50 \% \mathrm{Cl} / \mathrm{Br}$, with trace colors reflecting the color of emission. All absorption and photoluminescence measurements were taken in solution phase (toluene). 
halide compositions is a viable solution. We started with precursor mixtures which were used to produce $n=1$ or $n=2$ nanoplatelets of pure chloride, bromide, or iodide composition. We then mixed these solutions to make mixed halide nanoplatelets of $\mathrm{Cl} / \mathrm{Br}$ or $\mathrm{Br} / \mathrm{I}$ composition, in $10 \%$ increments, and synthesized nanoplatelets according to the same procedure described previously. In Figure $7 \mathrm{a}$, we show the absorption spectra for $n=1$ and $n=2$ nanoplatelets $(\mathrm{A}=\mathrm{FA}, \mathrm{B}=\mathrm{Pb})$, highlighting the excellent tunability that can be achieved using this strategy. In total, the absorption peak of the $n=1$ nanoplatelets can be tuned from 2.5 to $3.7 \mathrm{eV}$, and the $n=2$ peak can be tuned from 2.2 to $3.5 \mathrm{eV}$. All peaks for the mixed halide compositions are single peaks; however, we do note a broadening of the mixed halide peaks as compared to their pure counterparts-particularly in the $n=2$ samples.

In Figure $7 b$, we plot the energy of the absorption peak as a function of the halide composition. The solid lines are drawn between the pure halide compositions to evaluate how closely the experimental data follow a linear trend between the absorption peak of the pure compositions $(100 \% \mathrm{Cl}, 100 \% \mathrm{Br}$, $100 \%$ I). For $n=1$ nanoplatelets, we see that the absorption peak of halide mixtures closely follows its expected position based on the relative ratio of halide ions. The $n=2$ nanoplatelets also follow the expected trend, but we observe some deviation from ideal behavior for bromide content between 60 and $80 \%$ in $\mathrm{Cl} / \mathrm{Br}$ mixtures and for iodide content between 40 and $90 \%$ in $\mathrm{Br} / \mathrm{I}$ mixtures. It has been shown by Eperon et al. that bulk $\mathrm{FAPb}\left(\mathrm{Br}_{1-x} \mathrm{I}_{x}\right)_{3}$ will not crystallize in all proportions, likely due to differences in the crystal structures adopted by the pure forms (cubic for bromide, tetragonal for iodide), specifically when iodine content is in the range of 50$70 \%$ of the total halide content. ${ }^{42,46}$ While we have observed nanoplatelet formation for all ratios of halide mixtures, the deviation in $n=2$ nanoplatelets from a linear trend may be a reflection of the behavior seen in bulk crystals.

In Figure $7 c$, we present the photoluminescence spectra for the $n=2$ nanoplatelets of mixed halide compositions. The trace colors reflect the color of emission from the corresponding nanoplatelets. All mixtures of $\mathrm{Br} / \mathrm{I}$ exhibit photoluminescence, although the emission from 10 to $30 \%$ I content nanoplatelets was weak with long low-energy tails. As noted earlier, these mixtures also exhibit broadened absorption spectra, and we take this as an indication that halide ions may not be homogeneously mixed within each nanoplatelet or the ion content may be inhomogeneous throughout the ensemble. All mixtures of $\mathrm{Cl} / \mathrm{Br}$ which were within our detection limit showed strong photoluminescence. This corresponds to nanoplatelets with halide compositions between 10 and $50 \%$ Cl. Emission was also present from some of the mixed $\mathrm{Br} / \mathrm{I} n=$ 1 nanoplatelets, specifically in the range of 70-90\% I (see Figure S14) but was generally weak. Emission from $n=1$ nanoplatelets with mixed $\mathrm{Cl} / \mathrm{Br}$ composition was beyond our detection range. Values from Figure 7 are available in Tables S2 and S3.

\section{CONCLUSIONS}

Perovskite nanoplatelets, particularly those of $n=1$ and $n=2$ thickness, show promise as a highly tunable material system. The cation (A), metal (B), and halide (X) components can be altered or mixed in many compositions to achieve a desired absorption and emission energy with excellent specificity. The ability to tune the thickness $(n)$ is an additional dimension of flexibility over their bulk counterparts. The nanoplatelets benefit from a facile, room-temperature synthesis, efficient luminescence, and narrow absorption and emission properties for most compositions. Their large lateral dimensions will permit studies of energy transfer between the nanoplatelets and other materials, such as quantum dots, and the fabrication of electronic devices. Challenges related to large-scale synthesis, long-term nanoplatelet stability, and improved understanding of the nanoplatelet formation mechanism ${ }^{57}$ must be addressed in future studies. We note that tin-based nanoplatelets, if improved to be more air-stable, are especially promising as halide compositional tuning would permit the entire visible spectrum to be covered in a single, heavy-metal-free platform. ${ }^{17,58}$ Overall, colloidal perovskite nanoplatelets represent an exciting class of solution-processable materials for tunable light absorption and emission.

\section{EXPERIMENTAL METHODS}

Chemicals. When possible, chemicals were purchased from commercial suppliers (see Supporting Information). However, several of the ligand salts (LX) and cation salts (AX) used for perovskite synthesis are not commercially available and were synthesized inhouse. In general, this was done by reacting the amine species or the cation salt with a slight excess of the corresponding acid, followed by thorough washing with diethyl ether and recrystallization to purify the compound. For example, octylammonium iodide was synthesized by reacting $120 \mathrm{mmol}$ of octylamine with $130 \mathrm{mmol}$ of hydriodic acid in $100 \mathrm{~mL}$ of ethanol. The products were dried using rotary evaporation, washed thoroughly with diethyl ether, and recrystallized once using acetone to produce a white, shiny solid. All chemicals were stored in an oxygen- and water-free glovebox. Full details for the synthesis of each chemical used in this study are provided in the Supporting Information and Table S1.

Abbreviations. For ease of writing nanoplatelet compositions, we use the following abbreviations throughout to represent different chemical species. A: cesium $=\mathrm{Cs}$, formamidinium $=\mathrm{FA}$, methylammonium $=\mathrm{MA}$. $\mathrm{B}$ : lead $=\mathrm{Pb}$, tin $=\mathrm{Sn}$. $\mathrm{X}$ : chloride $=\mathrm{Cl}$, bromide $=$ $\mathrm{Br}$, iodide $=\mathrm{I}$. L: butylammonium $=\mathrm{BA}$, octylammonium $=\mathrm{OA}$.

Nanoplatelet Synthesis. Nanoplatelets were synthesized using a nonsolvent crystallization method. ${ }^{8,11,42}$ Syntheses were performed under ambient laboratory conditions, except for those involving tin, which were performed in a glovebox. Stock solutions were prepared by dissolving precursor salts $\left(\mathrm{AX}, \mathrm{BX}_{2}, \mathrm{LX}\right)$ in $\mathrm{DMF}$, typically at concentrations of $0.1 \mathrm{M}$. The stock solutions were then mixed in proper proportions to obtain either $n=1$ or $n=2$ nanoplatelets. For ease of calculating the mixing ratios, the formula for the nanoplatelets can be rewritten in terms of their precursor salts: $(\mathrm{LX})_{2}\left(\mathrm{BX}_{2}\right)_{n}(\mathrm{AX})_{n-1}$. Hence, for $n=1$ nanoplatelets, the stoichiometry calls for a ratio of 2:1:0 $\left(\mathrm{LX} / \mathrm{BX}_{2} / \mathrm{AX}\right)$ and a ratio of 2:2:1 for $n=2$ nanoplatelets. However, in this study, we used an excess of ligands for the $n=2$ nanoplatelets (typically 10:2:1 $\mathrm{LX} / \mathrm{BX}_{2} / \mathrm{AX}$ ) to achieve better thickness homogeneity and colloidal stability (see Figure S8).

In all cases, we have used a 50/50 mixture of octylammonium and butylammonium as the ligand species $\mathrm{L}$, which results in better thickness homogeneity. We hypothesize that octylammonium alone may be too bulky to bind to every site on the nanoplatelet surface, and so the addition of butylammonium can help to better passivate the nanoplatelets and prevent growth to thicker nanoplatelets. For example, the precursor mixture for the synthesis of $\mathrm{L}_{2}\left[\mathrm{FAPbBr}_{3}\right] \mathrm{PbBr}_{4}$ was 5 parts octylamonium bromide, 5 parts butylammonium bromide, 2 parts lead bromide, and 1 part formamidinium bromide.

The precursor solution was added dropwise to toluene undergoing vigorous stirring at room temperature (see Figure S1). The nanoplatelets form immediately, as evidenced by the appearance of photoluminescence (see Movie S1). For this study, a single drop of precursor solution $(\sim 10 \mu \mathrm{L})$ was added to $10 \mathrm{~mL}$ of toluene. Larger quantities of nanoplatelets can be produced by adding additional precursor drops or using higher concentration precursor stock solutions (such as 0.5 or $1.0 \mathrm{M}$ ). However, dropwise precursor 
addition can slightly red shift the emission peak with each drop, so we used a single precursor addition throughout this work. See the Supporting Information for additional details related to synthesis.

Postsynthesis Processing. The majority of data presented were collected in solution phase, using as-synthesized nanoplatelets in toluene. Nanoplatelets could be isolated from the toluene/DMF growth solution by centrifugation. Typically, the nanoplatelets in solution were centrifuged at $4000 \mathrm{rpm}$ for $5 \mathrm{~min}$ or longer, leading to at least partial precipitation. The nanoplatelets can then be redispersed in organic solvents and drop-cast into thin films (see Figure S2). We note that this process typically reduces the emission brightness of the nanoplatelets, which may be due to a loss of ligand coverage or exposure to oxygen and water when in the solid state. See the Supporting Information for a discussion of challenges related to nanoplatelet stability.

Characterization. Photoluminescence and absorption spectra were recorded using an Avantes fiber-optic spectrometer and Cary $5000 \mathrm{UV}-$ vis spectrophotometer, respectively. Samples were excited by a $365 \mathrm{~nm}$ fiber-coupled LED (Thorlabs) for photoluminescence measurements. Before analysis, photoluminescence spectra were converted to an energy scale using the procedure outlined by Mooney and Kambhampati. ${ }^{59}$ TEM was performed on a JEOL 2011 operating at $200 \mathrm{kV}$. TEM samples were prepared by centrifuging the reaction products to precipitate the nanoplatelets, redispersing in a small quantity of toluene $(\sim 250 \mu \mathrm{L})$, and drop-casting onto a carbon film TEM grid. XRD was performed using a PANanlytical X'Pert PRO operating at $45 \mathrm{kV}$ and $40 \mathrm{~mA}$ using a copper radiation source. XRD samples were prepared by centrifuging reaction products (made using $0.5 \mathrm{M}$ stock solutions), redispersing in a small quantity of toluene $(\sim 30 \mu \mathrm{L})$, and drop-casting onto glass slides. Tin-based samples were measured using an air-free sample holder. Substrate background scatter was subtracted from XRD patterns using HighScore Plus software. PLQY measurements were performed using an integrating sphere and CCD spectrograph (Princeton Instruments). ${ }^{60}$ Samples were excited using a $405 \mathrm{~nm}$ laser (PicoQuant). The relative efficiency of the detection system was determined using a calibrated tungsten halogen lamp. All samples had optical densities below 0.1 at $405 \mathrm{~nm}$ for these measurements, except for the tin-based perovskites, which transformed into an undesired phase if diluted (see Supporting Information) and so were measured with optical densities $>1$.

\section{ASSOCIATED CONTENT}

\section{S Supporting Information}

The Supporting Information is available free of charge on the ACS Publications website at DOI: 10.1021/acsnano.6b03496.

Detailed synthesis procedures, precursor preparation, stability discussion, TEM images, XRD patterns, and absorption/photoluminescence spectra (PDF) Movie S1 (MPG)

\section{AUTHOR INFORMATION}

\section{Corresponding Author}

*E-mail: tisdale@mit.edu.

\section{Notes}

The authors declare no competing financial interest.

\section{ACKNOWLEDGMENTS}

The authors thank Charles Settens for assistance with XRD data analysis and simulation, Thomas Mahony for assistance with PLQY measurements, Seung Kyun Ha for experimental assistance, and Daniel Congreve and Sandeep Pathak for valuable discussions. This work was supported as part of the Center for Excitonics, an Energy Frontier Research Center funded by the U.S. Department of Energy, Office of Science, Office of Basic Energy Sciences under Award DE-SC0001088 (MIT). This work made use of the MRSEC Shared
Experimental Facilities at MIT, supported by the National Science Foundation under Award Number DMR-08-19762. S.D.S. has received funding from the People Programme (Marie Curie Actions) of the European Union's Seventh Framework Programme (FP7/2007-2013) under REA Grant Agreement Number PIOF-GA-2013-622630.

\section{REFERENCES}

(1) Stranks, S. D.; Eperon, G. E.; Grancini, G.; Menelaou, C.; Alcocer, M. J. P.; Leijtens, T.; Herz, L. M.; Petrozza, A.; Snaith, H. J. Electron-Hole Diffusion Lengths Exceeding 1 Micrometer in an Organometal Trihalide Perovskite Absorber. Science 2013, 342, 341344

(2) Xing, G.; Mathews, N.; Sun, S.; Lim, S. S.; Lam, Y. M.; Grätzel, M.; Mhaisalkar, S.; Sum, T. C. Long-Range Balanced Electron- and Hole-Transport Lengths in Organic-Inorganic $\mathrm{CH} 3 \mathrm{NH} 3 \mathrm{PbI} 3$. Science 2013, 342, 344-347.

(3) Green, M. A.; Ho-Baillie, A.; Snaith, H. J. The Emergence of Perovskite Solar Cells. Nat. Photonics 2014, 8, 506-514.

(4) De Wolf, S.; Holovsky, J.; Moon, S.; Löper, P.; Niesen, B.; Ledinsky, M.; Haug, F.; Yum, J.; Ballif, C. Organometallic Halide Perovskites: Sharp Optical Absorption Edge and Its Relation to Photovoltaic Performance. J. Phys. Chem. Lett. 2014, 5, 1035-1039.

(5) Shi, D.; Adinolfi, V.; Comin, R.; Yuan, M.; Alarousu, E.; Buin, A.; Chen, Y.; Hoogland, S.; Rothenberger, A.; Katsiev, K.; Losovyj, Y.; Zhang, X.; Dowben, P. A.; Mohammed, O. F.; Sargent, E. H.; Bakr, O. M. Low Trap-State Density and Long Carrier Diffusion in Organolead Trihalide Perovskite Single Crystals. Science 2015, 347, 519-522.

(6) Stranks, S. D.; Snaith, H. J. Metal-Halide Perovskites for Photovoltaic and Light-Emitting Devices. Nat. Nanotechnol. 2015, 10, 391-402.

(7) Pazos-Outón, L. M.; Szumilo, M.; Lamboll, R.; Richter, J. M.; Crespo-Quesada, M.; Abdi-Jalebi, M.; Beeson, H. J.; Vrućinić, M.; Alsari, M.; Snaith, H. J.; Ehrler, B.; Friend, R. H.; Deschler, F. Photon Recycling in Lead Iodide Perovskite Solar Cells. Science 2016, 351, $1430-1433$.

(8) Schmidt, L. C.; Pertegas, A.; González-Carrero, S.; Malinkiewicz, O.; Agouram, S.; Espallargas, G. M.; Bolink, H. J.; Galian, R. E.; PérezPrieto, J. Nontemplate Synthesis of $\mathrm{CH} 3 \mathrm{NH} 3 \mathrm{PbBr} 3$ Perovskite Nanoparticles. J. Am. Chem. Soc. 2014, 136, 850-853.

(9) Dou, L.; Wong, A. B.; Yu, Y.; Lai, M.; Kornienko, N.; Eaton, S. W.; Fu, A.; Bischak, C. G.; Ma, J.; Ding, T.; Ginsberg, N. S.; Wang, L.W.; Alivisatos, A. P.; Yang, P. Atomically Thin Two-Dimensional Organic-Inorganic Hybrid Perovskites. Science 2015, 349, 1518-1521.

(10) Tyagi, P.; Arveson, S. M.; Tisdale, W. A. Colloidal Organohalide Perovskite Nanoplatelets Exhibiting Quantum Confinement. J. Phys. Chem. Lett. 2015, 6, 1911-1916.

(11) Sichert, J. A.; Tong, Y.; Mutz, N.; Vollmer, M.; Fischer, S.; Milowska, K. Z.; Cortadella, R. G.; Nickel, B.; Cardenas-Daw, C.; Stolarczyk, J. K.; Urban, A. S.; Feldmann, J. Quantum Size Effect in Organometal Halide Perovskite Nanoplatelets. Nano Lett. 2015, 15, 6521-6527.

(12) Protesescu, L.; Yakunin, S.; Bodnarchuk, M. I.; Krieg, F.; Caputo, R.; Hendon, C. H.; Yang, R. X.; Walsh, A.; Kovalenko, M. V. Nanocrystals of Cesium Lead Halide Perovskites (CsPbX3, X $=\mathrm{Cl}, \mathrm{Br}$, and I): Novel Optoelectronic Materials Showing Bright Emission with Wide Color Gamut. Nano Lett. 2015, 15, 3692-3696.

(13) Akkerman, Q. A.; D’Innocenzo, V.; Accornero, S.; Scarpellini, A.; Petrozza, A.; Prato, M.; Manna, L. Tuning the Optical Properties of Cesium Lead Halide Perovskite Nanocrystals by Anion Exchange Reactions. J. Am. Chem. Soc. 2015, 137, 10276-10281.

(14) Nedelcu, G.; Protesescu, L.; Yakunin, S.; Bodnarchuk, M. I.; Grotevent, M. J.; Kovalenko, M. V. Fast Anion-Exchange in Highly Luminescent Nanocrystals of Cesium Lead Halide Perovskites (CsPbX3, X = Cl, Br, I). Nano Lett. 2015, 15, 5635-5640.

(15) Zhang, F.; Zhong, H.; Chen, C.; Wu, X.; Hu, X.; Huang, H.; Han, J.; Zou, B.; Dong, Y. Brightly Luminescent and Color-Tunable 
Colloidal CH3NH3PbX3 (X = Br, I, Cl) Quantum Dots: Potential Alternatives for Display Technology. ACS Nano 2015, 9, 4533-4542.

(16) Sun, S.; Yuan, D.; Xu, Y.; Wang, A.; Deng, Z. Ligand-Mediated Synthesis of Shape-Controlled Cesium Lead Halide Perovskite Nanocrystals Via Reprecipitation Process at Room Temperature. ACS Nano 2016, 10, 3648-3657.

(17) Papavassiliou, G. C. Three- and Low-Dimensional Inorganic Semiconductors. Prog. Solid State Chem. 1997, 25, 125-270.

(18) Papavassiliou, G. C.; Koutselas, I. B. Structural, Optical and Related Properties of Some Natural Three- and Lower-Dimensional Semiconductor Systems. Synth. Met. 1995, 71, 1713-1714.

(19) Ishihara, T.; Takahashi, J.; Goto, T. Optical Properties Due to Electronic Transitions in Two-Dimensional Semiconductors $(\mathrm{CnH} 2 \mathrm{n}$ +1NH3)2PbI4. Phys. Rev. B: Condens. Matter Mater. Phys. 1990, 42, 11099-11107.

(20) Mitzi, D. B. Templating and Structural Engineering in OrganicInorganic Perovskites. J. Chem. Soc., Dalton Trans. 2001, 1-12.

(21) Mitzi, D. B.; Chondroudis, K.; Kagan, C. R. Organic-Inorganic Electronics. IBM J. Res. Dev. 2001, 45, 29-45.

(22) Mitzi, D. B. Synthesis, Structure, and Properties of OrganicInorganic Perovskites and Related Materials. Prog. Inorg. Chem. 1999, 48, $1-121$.

(23) Kamminga, M. E.; Fang, H.-H.; Filip, M. R.; Giustino, F.; Baas, J.; Blake, G. R.; Loi, M. A.; Palstra, T. T. M. Confinement Effects in Low-Dimensional Lead Iodide Perovskite Hybrids. Chem. Mater. 2016, $28,4554$.

(24) Yuan, Z.; Shu, Y.; Xin, Y.; Ma, B. Highly Luminescent Nanoscale Quasi-2D Layered Lead Bromide Perovskites with Tunable Emissions. Chem. Commun. 2016, 52, 3887-3890.

(25) Lignos, I.; Stavrakis, S.; Nedelcu, G.; Protesescu, L.; deMello, A. J.; Kovalenko, M. V. Synthesis of Cesium Lead Halide Perovskite Nanocrystals in a Droplet-Based Microfluidic Platform: Fast Parametric Space Mapping. Nano Lett. 2016, 16, 1869-1877.

(26) Yuan, Z.; Shu, Y.; Tian, Y.; Xin, Y.; Ma, B. A Facile One-Pot Synthesis of Deep Blue Luminescent Lead Bromide Perovskite Microdisks. Chem. Commun. 2015, 51, 16385-16388.

(27) Quan, L. N.; Yuan, M.; Comin, R.; Voznyy, O.; Beauregard, E. M.; Hoogland, S.; Buin, A.; Kirmani, A. R.; Zhao, K.; Amassian, A.; Kim, D. H.; Sargent, E. H. Ligand-Stabilized Reduced-Dimensionality Perovskites. J. Am. Chem. Soc. 2016, 138, 2649-2655.

(28) Tsai, H.; Nie, W.; Blancon, J.; Stoumpos, C. C.; Asadpour, R.; Harutyunyan, B.; Neukirch, A. J.; Verduzco, R.; Crochet, J. J.; Tretiak, S.; Pedesseau, L.; Even, J.; Alam, M. A.; Gupta, G.; Lou, J.; Ajayan, P. M.; Bedzyk, M. J.; Kanatzidis, M. G.; Mohite, A. D. High-Efficiency Two-Dimensional Ruddlesden-Popper Perovskite Solar Cells. Nature 2016, DOI: $10.1038 /$ nature 18306 .

(29) Yuan, M.; Quan, L. N.; Comin, R.; Walters, G.; Sabatini, R.; Voznyy, O.; Hoogland, S.; Zhao, Y.; Beauregard, E. M.; Kanjanaboos, P.; Lu, Z.; Kim, D. H.; Sargent, E. H. Perovskite Energy Funnels for Efficient Light-Emitting Diodes. Nat. Nanotechnol. 2016, DOI: $10.1038 /$ nnano.2016.110.

(30) Liang, D.; Peng, Y.; Fu, Y.; Shearer, M. J.; Zhang, J.; Zhai, J.; Zhang, Y.; Hamers, R. J.; Andrew, T. L.; Jin, S. Color-Pure VioletLight-Emitting Diodes Based on Layered Lead Halide Perovskite Nanoplates. ACS Nano 2016, 10, 6897.

(31) Akkerman, Q. A.; Motti, S. G.; Srimath Kandada, A. R.; Mosconi, E.; D’Innocenzo, V.; Bertoni, G.; Marras, S.; Kamino, B. A.; Miranda, L.; De Angelis, F.; Petrozza, A.; Prato, M.; Manna, L. Solution Synthesis Approach to Colloidal Cesium Lead Halide Perovskite Nanoplatelets with Monolayer-Level Thickness Control. J. Am. Chem. Soc. 2016, 138, 1010-1016.

(32) Bekenstein, Y.; Koscher, B. A.; Eaton, S. W.; Yang, P.; Alivisatos, A. P. Highly Luminescent Colloidal Nanoplates of Perovskite Cesium Lead Halide and Their Oriented Assemblies. J. Am. Chem. Soc. 2015, 137, 16008-16011.

(33) Lv, L.; Xu, Y.; Fang, H.; Luo, W.; Xu, F.; Liu, L.; Wang, B.; Zhang, X.; Yang, D.; Hu, W.; Dong, A. Generalized Colloidal Synthesis of High-Quality, Two-Dimensional Cesium Lead Halide Perovskite
Nanosheets and Their Applications in Photodetectors. Nanoscale 2016, 8, 13589

(34) Shamsi, J.; Dang, Z.; Bianchini, P.; Canale, C.; Di Stasio, F.; Brescia, R.; Prato, M.; Manna, L. Colloidal Synthesis of Quantum Confined Single Crystal CsPbBr3 Nanosheets with Lateral Size Control up to the Micrometer Range. J. Am. Chem. Soc. 2016, 138, $7240-7243$

(35) Vybornyi, O.; Yakunin, S.; Kovalenko, M. V. Polar-Solvent-Free Colloidal Synthesis of Highly Luminescent Alkylammonium Lead Halide Perovskite Nanocrystals. Nanoscale 2016, 8, 6278-6283.

(36) Wu, X.; Trinh, M. T.; Zhu, X.-Y. Excitonic Many-Body Interactions in Two-Dimensional Lead Iodide Perovskite Quantum Wells. J. Phys. Chem. C 2015, 119, 14714-14721.

(37) Hassan, Y.; Song, Y.; Pensack, R. D.; Abdelrahman, A. I.; Kobayashi, Y.; Winnik, M. A.; Scholes, G. D. Structure-Tuned Lead Halide Perovskite Nanocrystals. Adv. Mater. 2016, 28, 566-573.

(38) Dou, L. T.; Wong, A. B.; Yu, Y.; Lai, M. L.; Kornienko, N.; Eaton, S. W.; Fu, A.; Bischak, C. G.; Ma, J.; Ding, T. N.; Ginsberg, N. S.; Wang, L. W.; Alivisatos, A. P.; Yang, P. D. Atomically Thin TwoDimensional Organic-Inorganic Hybrid Perovskites. Science 2015, 349, $1518-1521$

(39) Sapori, D.; Kepenekian, M.; Pedesseau, L.; Katan, C.; Even, J. Quantum Confinement and Dielectric Profiles of Colloidal Nanoplatelets of Halide Inorganic and Hybrid Organic-Inorganic Perovskites. Nanoscale 2016, 8, 6369-6378.

(40) Goldschmidt, V. M. The Laws of Crystal Chemistry. Naturwissenschaften 1926, 14, 477-485.

(41) Kieslich, G.; Sun, S.; Cheetham, A. K. Solid-State Principles Applied to Organic-Inorganic Perovskites: New Tricks for an Old Dog. Chem. Sci. 2014, 5, 4712-4715.

(42) Pathak, S.; Sakai, N.; Rivarola, F. W. R.; Stranks, S. D.; Liu, J.; Eperon, G. E.; Ducati, C.; Wojciechowski, K.; Griffiths, J. T.; Haghighirad, A. A.; Pellaroque, A.; Friend, R. H.; Snaith, H. J. Perovskite Crystals for Tunable White Light Emission. Chem. Mater. 2015, 27, 8066-8075.

(43) Even, J.; Pedesseau, L.; Katan, C. Understanding Quantum Confinement of Charge Carriers in Layered 2D Hybrid Perovskites. ChemPhysChem 2014, 15, 3733-3741.

(44) Umebayashi, T.; Asai, K.; Kondo, T.; Nakao, A. Electronic Structures of Lead Iodide Based Low-Dimensional Crystals. Phys. Rev. B: Condens. Matter Mater. Phys. 2003, 67, 155405.

(45) Stoumpos, C. C.; Kanatzidis, M. G. The Renaissance of Halide Perovskites and Their Evolution as Emerging Semiconductors. Acc. Chem. Res. 2015, 48, 2791-2802.

(46) Eperon, G. E.; Stranks, S. D.; Menelaou, C.; Johnston, M. B.; Herz, L. M.; Snaith, H. J. Formamidinium Lead Trihalide: A Broadly Tunable Perovskite for Efficient Planar Heterojunction Solar Cells. Energy Environ. Sci. 2014, 7, 982-988.

(47) Noel, N. K.; Stranks, S. D.; Abate, A.; Wehrenfennig, C.; Guarnera, S.; Haghighirad, A.; Sadhanala, A.; Eperon, G. E.; Pathak, S. K.; Johnston, M. B.; Petrozza, A.; Herz, L. M.; Snaith, H. J. Lead-Free Organic-Inorganic Tin Halide Perovskites for Photovoltaic Applications. Energy Environ. Sci. 2014, 7, 3061-3068.

(48) Jellicoe, T. C.; Richter, J. M.; Glass, H. F. J.; Tabachnyk, M.; Brady, R.; Dutton, S. E.; Rao, A.; Friend, R. H.; Credgington, D.; Greenham, N. C.; Bohm, M. L. Synthesis and Optical Properties of Lead-Free Cesium Tin Halide Perovskite Nanocrystals. J. Am. Chem. Soc. 2016, 138, 2941-2944.

(49) Comin, R.; Walters, G.; Thibau, E. S.; Voznyy, O.; Lu, Z. H.; Sargent, E. H. Structural, Optical, and Electronic Studies of WideBandgap Lead Halide Perovskites. J. Mater. Chem. C 2015, 3, 88398843.

(50) Kulbak, M.; Cahen, D.; Hodes, G. How Important Is the Organic Part of Lead Halide Perovskite Photovoltaic Cells? Efficient CsPbBr3 Cells. J. Phys. Chem. Lett. 2015, 6, 2452-2456.

(51) Yantara, N.; Bhaumik, S.; Yan, F.; Sabba, D.; Dewi, H. A.; Mathews, N.; Boix, P. P.; Demir, H. V.; Mhaisalkar, S. Inorganic Halide Perovskites for Efficient Light-Emitting Diodes. J. Phys. Chem. Lett. 2015, 6, 4360-4364. 
(52) Hanusch, F. C.; Wiesenmayer, E.; Mankel, E.; Binek, A.; Angloher, P.; Fraunhofer, C.; Giesbrecht, N.; Feckl, J. M.; Jaegermann, W.; Johrendt, D.; Bein, T.; Docampo, P. Efficient Planar Heterojunction Perovskite Solar Cells Based on Formamidinium Lead Bromide. J. Phys. Chem. Lett. 2014, 5, 2791-2795.

(53) Eperon, G. E.; Beck, C. E.; Snaith, H. J. Cation Exchange for Thin Film Lead Iodide Perovskite Interconversion. Mater. Horiz. 2016, 3, 63-71.

(54) Kong, W.; Ye, Z.; Qi, Z.; Zhang, B.; Wang, M.; Rahimi-Iman, A.; $\mathrm{Wu}, \mathrm{H}$. Characterization of an Abnormal Photoluminescence Behavior Upon Crystal-Phase Transition of Perovskite CH3NH3PbI3. Phys. Chem. Chem. Phys. 2015, 17, 16405-16411.

(55) Koh, T. M.; Krishnamoorthy, T.; Yantara, N.; Shi, C.; Leong, W. L.; Boix, P. P.; Grimsdale, A. C.; Mhaisalkar, S. G.; Mathews, N. Formamidinium Tin-Based Perovskite with Low Eg for Photovoltaic Applications. J. Mater. Chem. A 2015, 3, 14996-15000.

(56) Stoumpos, C. C.; Malliakas, C. D.; Kanatzidis, M. G. Semiconducting Tin and Lead Iodide Perovskites with Organic Cations: Phase Transitions, High Mobilities, and Near-Infrared Photoluminescent Properties. Inorg. Chem. 2013, 52, 9019-9038.

(57) Riedinger, A.; Ott, F. D.; Mule, A.; Mazzotti, S.; Knuesel, P. N.; Kress, S. J. P.; Prins, F.; Erwin, S. C.; Norris, D. J. How Semiconductor Nanoplatelets Form. arXiv:1605.06553, 2016.

(58) Peedikakkandy, L.; Bhargava, P. Composition Dependent Optical, Structural and Photoluminescence Characteristics of Cesium Tin Halide Perovskites. RSC Adv. 2016, 6, 19857-19860.

(59) Mooney, J.; Kambhampati, P. Get the Basics Right: Jacobian Conversion of Wavelength and Energy Scales for Quantitative Analysis of Emission Spectra. J. Phys. Chem. Lett. 2013, 4, 3316-3318.

(60) de Mello, J. C.; Wittmann, H. F.; Friend, R. H. An Improved Experimental Determination of External Photoluminescence Quantum Efficiency. Adv. Mater. 1997, 9, 230-232. 